\title{
Vision Sensor Calibration Method based on Flexible 3d Target and Invariance of Cross Ratio
}

\author{
Jingli Wei1, Zhiwei Liu1 \& Fangling Cheng1 \\ ${ }^{1}$ CSR Qingdao Sifang Co., Ltd, Qingdao, Shandong, 266111, China
}

\begin{abstract}
Keywords: Vision Sensor, Calibration, Flexible 3d Target, Invariance of Cross Ratio
\end{abstract}
\begin{abstract}
A vision sensor calibration method based on flexible three-dimensional (3d) target and invariance of cross ratio is proposed. Flexible $3 \mathrm{~d}$ target is obtained by moving planar target to different positions. According to the relative position relationship of planar targets in the different positions, unify target points to the base coordinate frame of the flexible $3 \mathrm{~d}$ target. Using the invariance of cross ratio, the coordinates of the points in the light plane are obtained, then converted to sensor measurement coordinate system. Finally, vision sensor calibration parameters are obtained by the nonlinear optimization algorithm. The experimental results demonstrate that the proposed method can obtain higher calibration accuracy, reduce the calibration cost. In addition, the proposed method can adapt to field calibration for vision system.
\end{abstract}

\section{Introduction}

Recently, 3D vision measuring equipment is widely used in reverse engineering, autonomous navigation and visual reality [1][2]. Most of 3d vision sensor measure methods use active vision measure technology, which is the structured light sensor emits a certain mode of structure light on the profile of the object, structured light image information is transferred to a computer, and then image features are extracted by image processing. Eventually, a set of pixel-to-pixel matching correspondence between camera and projector is set up, resulting in three-dimension profile measurement. Based on the different pattern of optical projector, the mode of structure light is divided into line structure light, point structure light, multi-line structure light and so on. However, line structure light is widely applied to industrial vision measuring fields due to its simple structure, small volume, light weight, low cost, convenient calibration, and high accuracy of measurement [3].

Currently, different approaches for calibrating projector have been proposed in many literatures. A moving one-dimensional (1D) target arbitrarily placed in the field of view to simplify the calibration procedure [4]. In Robert Dewar's method [5], several thin non-coplanar threads are strained in the space illuminated by a light stripe, and then several bright light dots are obtained as the control points whose 3D coordinates can be measured by means of a theodolite. However, this method requires complicated and expensive equipment. Huynh [6] has proposed a method, in which the world points on the light stripe plane are generated based on the invariance of the cross-ratio. In the method, a 3D calibration target is difficult to be manufactured accurately. Besides the above methods, there are other methods of calibrating a structured light vision system being presented in the literature [7]. Wei [8] has proposed a novel 1D target-based calibration method for structured light vision sensors by randomly viewing a $1 \mathrm{D}$ target from different unknown orientations positioned within the field of view.

This paper proposes a new calibration approach of vision system based on flexible 3d target and invariance of cross ratio. Flexible $3 \mathrm{~d}$ target is established. Then, control points in local coordinate frame are transferred to the base coordinate frame of the flexible 3d target by transformation matrix. Using the invariance of cross ratio, the coordinates of the points in the light planar are obtained, then converted to sensor measurement coordinate. Finally, vision system calibration parameters are obtained by the nonlinear optimization algorithm. The experimental results demonstrate that the proposed method can obtain higher calibration accuracy, reduce the calibration cost. In addition, the proposed method can adapt to field calibration for vision system. 


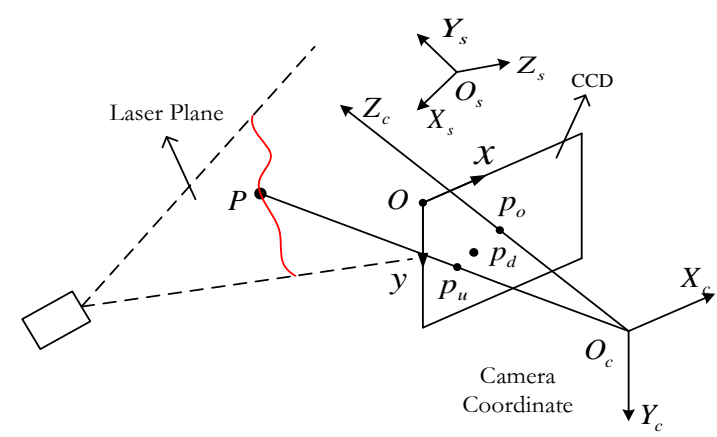

Figure1 Model of vision sensor

Figure 1 shows the line structured light model. Os-XsYsZs is the measuring-coordinate of vision sensor. The Origin Os, the axis OsXs and OsYs are on the laser plane, OsZs is normal to the laser plane. We set that the point $\mathrm{P}$ is on the laser stripe, Oc-XcYcZc is camera coordinate. $\mathrm{O}$-xy is the image plane. po is the principal point of the camera. The point pu is the ideal mapping point of $\mathrm{P}$. pd is the undistorted mapping point. [xd, $\mathrm{yd}]$ is the coordinate of $\mathrm{pd},[\mathrm{xu}, \mathrm{yu}]$ is the coordinate of pu, $[\mathrm{x} 0, \mathrm{y} 0]$ is the coordinate of po. The relationship between pu and pd is computed as follows:

where

$$
\begin{aligned}
& x_{u}=x_{d}+\overline{x_{d}}\left(k_{1} \cdot r_{d}^{2}+k_{2} \cdot r_{d}^{4}\right)+\left[p_{1} \cdot\left(r_{d}^{2}+2 \cdot \overline{x_{d}}\right)+2 p_{2} \overline{x_{d}} \overline{y_{d}}\right] \\
& y_{u}=y_{d}+\overline{y_{d}}\left(k_{1} \cdot r_{d}^{2}+k_{2} \cdot r_{d}^{4}\right)+\left[p_{2} \cdot\left(r_{d}^{2}+2 \cdot{\overline{y_{d}}}^{2}\right)+2 p_{1} \overline{x_{d} y_{d}}\right]
\end{aligned}
$$

$$
\overline{x_{d}}=x_{d}-x_{0}, \overline{y_{d}}=y_{d}-y_{0} ; r_{d}^{2}={\overline{x_{d}}}^{2}+{\overline{y_{d}}}^{2} .
$$

$\left[k_{1}, k_{2}, p_{1}, p_{2}\right]$ is the distortion coefficient of camera.

The relationship between $P$ and $p_{u}$ is as follows:

$$
\lambda \cdot \tilde{p}_{u}=K_{c} \cdot\left[\begin{array}{ll}
\boldsymbol{R} & \boldsymbol{T}
\end{array}\right] \cdot \tilde{P}
$$

where $\lambda$ is a scale factor, $\tilde{p}_{u}$ is the homogeneous coordinate of $p_{y}, \mathrm{R}$ is unitary orthogonal matrix, $\boldsymbol{T}$ is the translation vector, $\boldsymbol{K}_{\boldsymbol{c}}$ is the internal matrix of the camera.

By developing the formula (2), the following formula is obtained:

$$
\lambda \cdot\left[\begin{array}{c}
x_{u} \\
y_{u} \\
1
\end{array}\right]=\boldsymbol{H} \cdot\left[\begin{array}{c}
x_{s} \\
y_{s} \\
1
\end{array}\right]=\left[\begin{array}{lll}
h_{11} & h_{12} & h_{13} \\
h_{21} & h_{22} & h_{23} \\
h_{31} & h_{32} & h_{33}
\end{array}\right] \cdot\left[\begin{array}{c}
x_{s} \\
y_{s} \\
1
\end{array}\right]
$$

where $\lambda$ is a scale factor, $H$ is the parameter of the line structured-light.

\section{Flexible 3d Target theory}

\subsection{Flexible 3d Target model}

Figure 2 describes the established process of flexible 3d target.

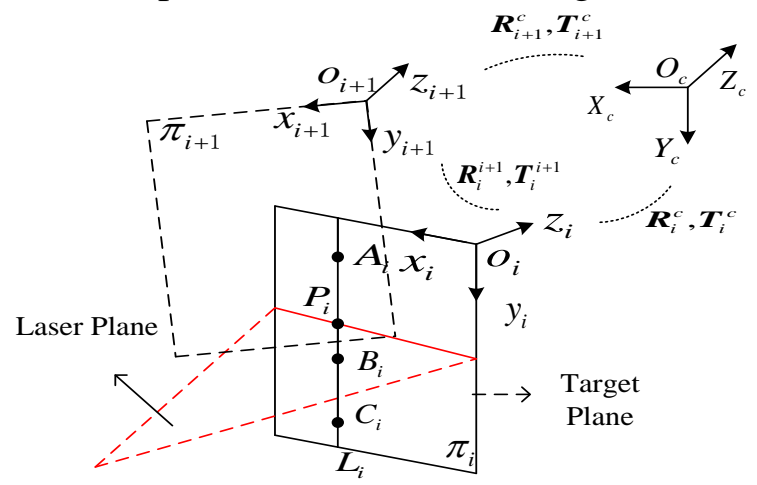

Figure 2 Flexible 3d target 
In order to determine the structure light plane, a flexible planar target method is given. Flexible $3 \mathrm{~d}$ target is formed by moving planar calibration board to different orientations, and the light stripe is projected onto the planar calibration board. We assume that the first target plane is the reference coordinate frame, then establish local coordinate system in every position of the target plane. The point $\mathrm{Pi}$ is the intersection of the co-line point and the stripe line in target plane. Then, the point $\mathrm{Pi}$ coordinate in local coordinate system is solved based on the invariance of cross ratio [9].

At different position of the target plane, we can resolve the relationship between the camera coordinate frame and the local coordinate frame of the target plane by the world to image co-ordinates, i.e. the camera external parameters. At the ith position of the target plane, the camera external parameters is rotation $R_{i}^{c}$ and translation $T_{i}^{c}$. However, the rotation $R_{i}^{i+1}$ and translation $T_{i}^{i+1}$ among the adjacent planar target are as follows:

$$
\begin{aligned}
& R_{i}^{i+1}=R_{i+1}^{c} \cdot R_{i}^{c} ; \\
& T_{i}^{i+1}=T_{i+1}^{c}-R_{i+1}^{c} \cdot R_{i}^{c} \cdot T_{i}^{c} ;
\end{aligned}
$$

The control point coordinates in local coordinate system are switched to reference coordinate frame via equation (4). So the established process of flexible $3 \mathrm{~d}$ target is done.

As can be seen above, the established process of flexible $3 \mathrm{~d}$ target is flexibility, reduces the cost of the calibration equipment and simplifies the calibrating procedure.

\subsection{Uniqueness sorting}

As shown in Figure 3(a), we use a specifically designed calibration board. In which, the center of every circle is the landmark point. In addition, there are five bigger circles to ensure the uniqueness of landmark order. According to the following principles, the fivcontrol pointe big control points are determined. The first one is that the distance of landmark point between number 2 and number 3 is the minimum, while the distance of control point between number 4 and number 5 is maximal. The second one is that the distance of control point between number 1 and number 3 is greater than that between number 1 and number 2 . The last one is the distance of control point between number 3 and number 4 is less than that between number 3 and number 5 .

After determining the order of the five control points, we can compute the homography matrix between pixel coordinate system and world coordinate system of calibration board. Then, the centers of other circles are detected using edge detection and ellipse fitting. The homography matrix is used to compute the image coordinate of control point. Finally, the real mapping points are calculated by minimizing the distance between the projective points and the ellipse center coordinates. Figure 3(b) shows the result of coded target points under an arbitrary position of the calibration board.

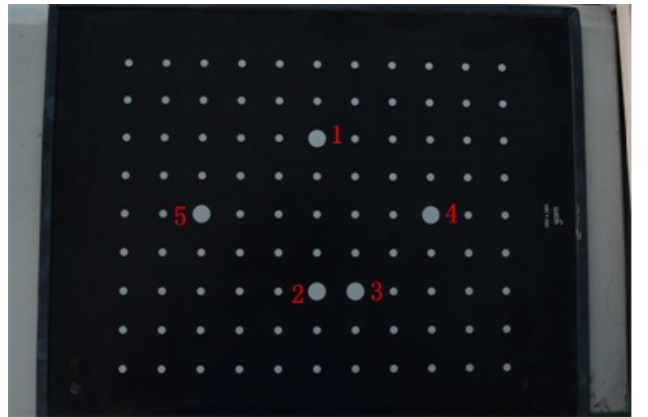

(a) The sorted control points

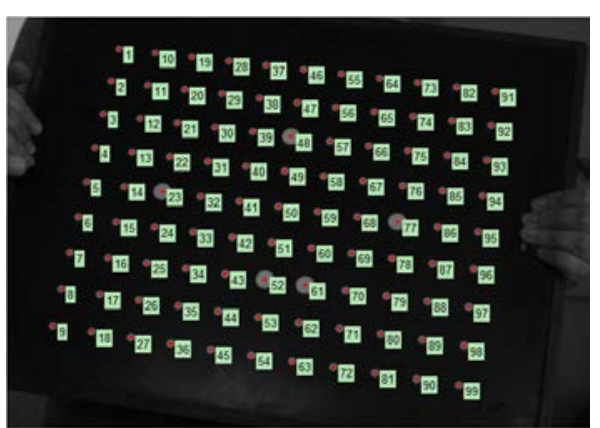

(b) The coded target point

Figure 3 The calibration board

\section{Calibration for structure light parameters}

\subsection{Transformation matrix among coordinate frames}

The mapping matrix $\mathrm{H}$ of the structure light is the transformation matrix from measuring coordinate to image plane. The transformation relation between the reference coordinate frame and the measuring coordinate frame is as follows: 


$$
\widetilde{P}_{s}=\boldsymbol{T}_{w}^{s} \cdot \widetilde{P}_{w}
$$

Where the points $\tilde{P}_{s}$ and $\tilde{P}_{w}$ are respectively the homogeneous coordinates of measuring coordinate and the reference coordinate, the transform matrix $\boldsymbol{T}_{w}^{c}$ is as follows:

$$
\boldsymbol{T}_{w}^{s}=\left[\begin{array}{cc}
\boldsymbol{R}_{w}^{s} & -\boldsymbol{R}_{w}^{s} \cdot \tilde{P}_{0} \\
\boldsymbol{0}^{T} & 1
\end{array}\right]
$$

Let us suppose that the normal vector of the light plane is $\boldsymbol{n}=\left[n_{1}, n_{2}, n_{3}\right]^{T}$, The direction vector of the measuring coordinate system is $\mathbf{z}_{\boldsymbol{w}}=[0,0,1]^{T}$. The rotation axis and rotation angle of the vector $\mathrm{n}$ and $\mathrm{zw}$ are respectively:

$$
\begin{aligned}
& \boldsymbol{a}=\left(\boldsymbol{n} \times \mathbf{z}_{\boldsymbol{w}}\right) /\left\|\boldsymbol{n} \times \mathbf{z}_{\boldsymbol{w}}\right\| ; \\
& \varphi=\arccos \left(\boldsymbol{n} \cdot \mathbf{z}_{\boldsymbol{w}}\right) ;
\end{aligned}
$$

The rotation matrix $R_{w}^{s}$ is expressed by as follows:

$$
\boldsymbol{R}_{w}^{s}=\boldsymbol{a} \cdot \boldsymbol{a}^{T}+\cos \varphi\left(\boldsymbol{I}-\boldsymbol{a} \cdot \boldsymbol{a}^{T}\right)+\sin \varphi(\boldsymbol{I} \times \boldsymbol{a})
$$

Where I is an unit matrix, $I \times a$ is the antisymmetric matrix.

\subsection{Optimizing the mapping matrix}

According to the control points in the light plane and image coordinates, the mapping matrix $\mathrm{H}$ is solved by formula (3).

The mapping matrix $\mathrm{H}$ is optimized by the nonlinear optimization method. Optimizing the object function is defined as follows:

$$
f(\boldsymbol{H})=\sum_{i}^{n u m}\left(m_{i}-\hat{m}_{i}\right)^{T}\left(m_{i}-\hat{m}_{i}\right)
$$

Where num is the number of control point, mi is image point. $\hat{m}_{i}$ is image point by mapping matrix $\mathrm{H}$ with the following expression:

$$
\hat{m}_{i}=\frac{1}{\boldsymbol{h}_{3}^{T}} \cdot\left[\begin{array}{l}
\boldsymbol{h}_{1}^{T} M_{i} \\
\boldsymbol{h}_{2}^{T} M_{i}
\end{array}\right]
$$

Where $\hat{h}_{i}(\mathrm{i}=1,2,3)$ is the ith row of the matrix $H, M_{i}$ is the point coordinate in measuring-coordinate.

Then, a nonlinear optimization function is established when the radial distortions are taken into consideration. Finally, the calibration is finished by using the Leveneberg-Marquardt (LM) algorithm.

\section{Experiment}

\subsection{Vision sensor calibration}

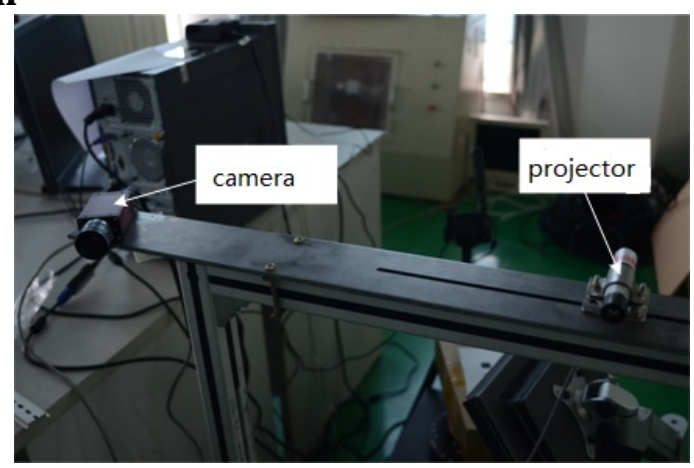

Figure 5 Vision sensor equipment

As shown in Fig 5, the vision sensor experiment equipment consists of a camera with an 8mm 
lens and a projector.

Firstly, camera calibration parameter, i.e. intrinsic matrix $\mathrm{KC}$ and distortion matrix kc are obtained by Zhang[10] method. The result shows that the reprojection error is 0.1522 pixel, with higher accuracy. Figure 6 shows image set for vision sensor calibration.

Then, we set the first image of the image set as a benchmark, then unify target landmark to the base coordinate frame of the flexible 3D target according to the relative position relationship of planar targets in the different positions.

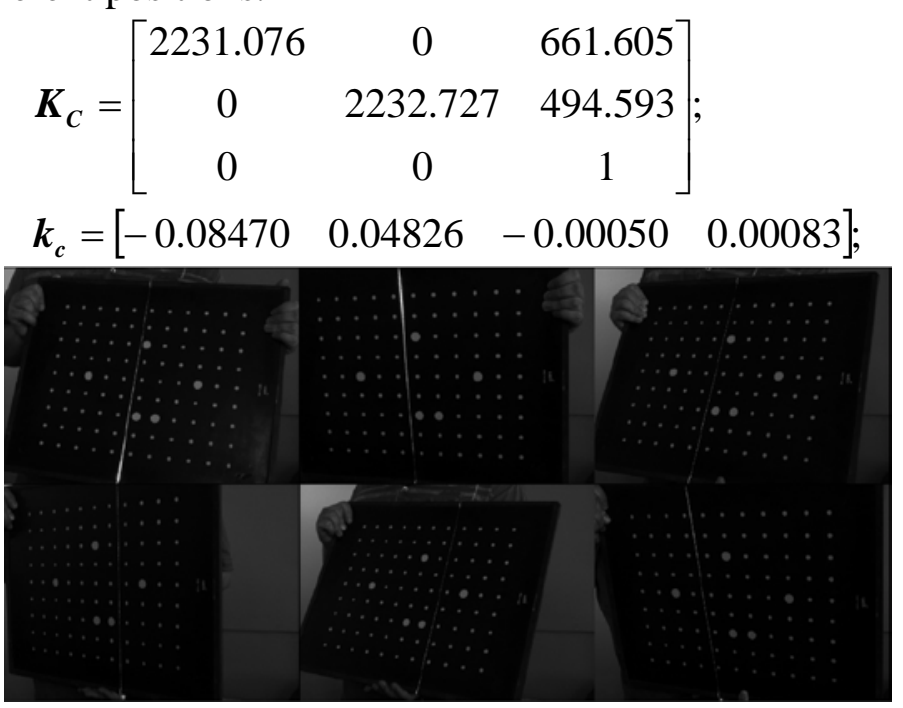

Figure 6 Image set for vision sensor calibration

Fig.7(a) shows the target points in reference coordinate, Fig.7(b) shows the Target points in measuring coordinate, Fig.7(a) shows the normalized image coordinate.

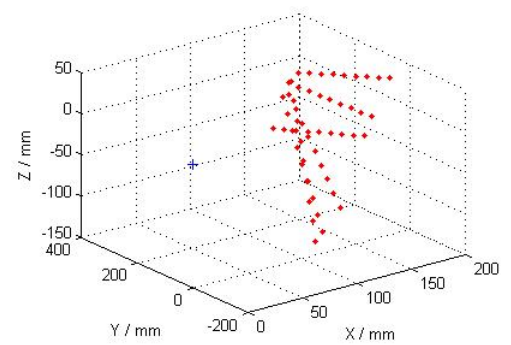

(a) points in reference coordinate

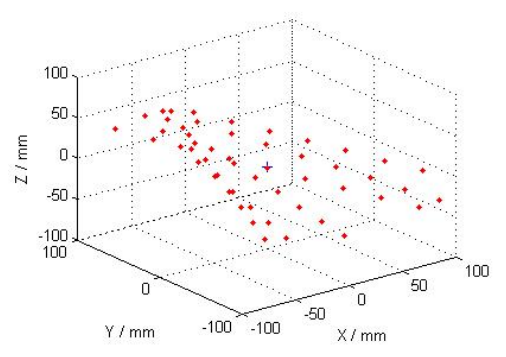

(b) points in measuring coordinate coordinate

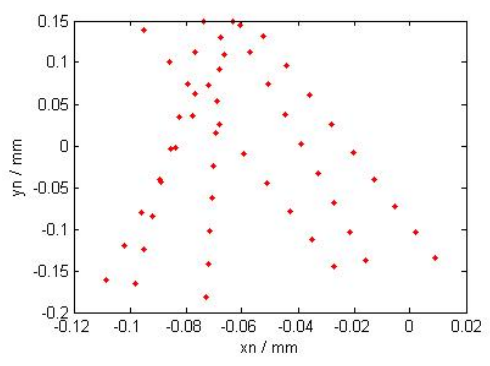

(c)Normalized image

Figure 7 3d coordinate of target points

Finally, the homography matrix $H$ between the light plane and image plane is obtained by equation (7).

$$
\boldsymbol{H}=\left[\begin{array}{ccc}
1825.3201 & 220.4005 & 94.8310 \\
-349.1362 & 981.9752 & -12.6930 \\
-1.7224 & -0.04176 & 1
\end{array}\right]
$$

\section{Accuracy assessment}

The absolute and relative error between the reference values and measured values are employed to evaluate vision sensor calibration accuracy. As is shown in Figure 1, normalized image coordinate, target coordinate and measuring coordinate are given. The absolute and relative errors of distance between the reference values and measured values are shown in Table 2, Sequence pairs represent the first target point and other target point. 
Table 1: Target point coordinate values

\begin{tabular}{ccccccc}
\hline \multirow{2}{*}{ Sequence } & \multicolumn{2}{c}{$\begin{array}{c}\text { Normalized image coordin } \\
\text { ate } / \mathrm{mm}\end{array}$} & \multicolumn{2}{c}{ Target coordinate } & \multicolumn{2}{c}{ Measuring coordinate } \\
& $x_{n}$ & $y_{n}$ & $X_{s}$ & $Y_{s}$ & $X_{s}$ & $Y_{s}$ \\
\hline 1 & -0.0589 & -0.1621 & 158.5291 & 0 & -43.7035 & -136.531 \\
& & & & & & 9 \\
2 & -0.0605 & -0.1237 & 158.1646 & 35 & -38.5623 & -101.877 \\
3 & -0.0620 & -0.0851 & 157.8626 & 70 & -33.4224 & -67.2313 \\
4 & -0.0636 & -0.0466 & 157.5114 & 105 & -28.2838 & -32.5938 \\
5 & -0.0651 & -0.0079 & 157.1460 & 140 & -23.1455 & 2.0413 \\
6 & -0.0667 & 0.0308 & 156.7978 & 175 & -18.0067 & 36.6802 \\
7 & -0.0682 & 0.0696 & 156.3863 & 210 & -12.8737 & 71.2793 \\
8 & -0.0698 & 0.1084 & 155.9595 & 245 & -7.7388 & 105.8918 \\
9 & -0.0713 & 0.1473 & 155.5889 & 280 & -2.6090 & 140.4699 \\
\hline
\end{tabular}

As shown in Table 2, the absolute error is less than $0.1 \mathrm{~mm}$. Meanwhile, relative error is less than $0.1 \%$. The experimental results demonstrate the proposed method can obtain higher calibration accuracy to meet detection requirements.

Table2: The error comparison between the reference values and measured values

\begin{tabular}{ccccc}
\hline $\begin{array}{c}\text { Sequence } \\
\text { pair }\end{array}$ & $\begin{array}{c}\text { Reference value } \\
\text { / mm }\end{array}$ & $\begin{array}{c}\text { Measured value } \\
\text { / mm }\end{array}$ & $\begin{array}{c}\text { Absolute error } \\
\text { / mm }\end{array}$ & $\begin{array}{c}\text { Relative erro } \\
\mathrm{r} / \%\end{array}$ \\
\hline$(1,2)$ & 35.0019 & 35.0341 & 0.0322 & 0.0920 \\
$(1,3)$ & 70.0032 & 70.0591 & 0.0559 & 0.0798 \\
$(1,4)$ & 105.0049 & 105.0756 & 0.0707 & 0.0673 \\
$(1,5)$ & 140.0068 & 140.0898 & 0.0830 & 0.0592 \\
$(1,6)$ & 175.0086 & 175.1078 & 0.0992 & 0.0567 \\
$(1.7)$ & 210.0109 & 210.0856 & 0.0747 & 0.0356 \\
$(1.8)$ & 245.0135 & 245.0769 & 0.0635 & 0.0259 \\
$(1,9)$ & 280.0154 & 280.0334 & 0.0180 & 0.0064 \\
\hline
\end{tabular}

\section{Conclusions}

A new calibration approach of vision system based on flexible 3d target and invariance of cross ratio is proposed. In the method, flexible $3 \mathrm{~d}$ target is established. The point coordinates in the light planar are obtained by using the invariance of cross ratio, then converted to sensor measuring coordinate. Finally, vision system calibration parameters are obtained by the nonlinear optimization function. The method generated large number of control points for vision sensor. The experimental results demonstrate that the proposed method can obtain higher calibration accuracy and reduce the calibration cost to adapt to field calibration.

\section{Acknowledgement}

The e-mail of the corresponding author is chenyueling@sia.cn.

\section{References}

[1]. Gao Y, Wang M, Ji R, Wu X, Dai Q. 3D object retrieval with hausdorff distance learning. IEEE Transactions on industrial electronics, 2014, 61: 2088-2098.

[2].Gao Y, Wang M, Tao D, Ji R, Dai Q. 3-d object retrieval and recognition with hypergraph analysis. IEEE Transactions on Image Processing, 2012, 21: 4290-4303.

[3].B Sandro, P Alessandro, RA Viviano, Three-dimensional point cloud alignment detecting 
fiducial markers by structured light stereo imaging. Mach Vis Appl, 2012, 23(2), 217-229.

[4]. Wei ZZ, Cao LJ, Zhang GJ. A novel 1D target-based calibration method with unknown orientation for structured light vision sensor. Optics and Laser Technology, 2010, 42(4): 570-4.

[5]. Dewar R. Self-generated targets for spatial calibration of structured light optical sectioning sensors with respect to an external coordinate system[C]. Robots and Vision'88 Conf. Proceedings, Detroit, 1988: 5-13.

[6].Huynh D Q. Calibration a structured light stripe system: a novel approach [J]. International Journal of Computer Vision, 1999, 33(1):73-86.

[7]. Xu J, Douet J, Zhao JG, Chen K. A simple calibration method for structured light-based 3D profile measurement. Optics \& Laser Technology, 2013, 48: 187-193.

[8]. Wei Z, Cao L, Zhang G. A novel 1D target-based calibration method with unknown orientation for structured light vision sensor. Optics \& Laser Technology, 2010, 42: 570-574.

[9].YQ Shi, CK Sun, BG Wang, P Wang, HX Duan, A global calibration method of multi-vision sensors in the measurement of engine cylinder joint surface holes. International Conference on Materials, Mechatronics and Automation. 2011, pp. 1182-1188.

[10]. Zhang Z, A flexible new technique for camera calibration [J]. IEEE Transactions on Pattern Analysis and Machine Intelligence, 2000, 22(11): 1330-1334. 